\title{
Optical design for the narrow field infrared adaptive optics system (NFIRAOS) petite on the thirty meter telescope
}

Brian J. Bauman, Donald T. Gavel, Richard G. Dekany, Brent L. Ellerbroek

Brian J. Bauman, Donald T. Gavel, Richard G. Dekany, Brent L. Ellerbroek, "Optical design for the narrow field infrared adaptive optics system (NFIRAOS) petite on the thirty meter telescope," Proc. SPIE 5903, Astronomical Adaptive Optics Systems and Applications II, 590303 (25 August 2005); doi: $10.1117 / 12.618220$

SPIE. Event: Optics and Photonics 2005, 2005, San Diego, California, United States 


\title{
Optical design for the Narrow Field InfraRed Adaptive Optics System (NFIRAOS) Petite on the Thirty Meter Telescope
}

\author{
Brian J. Bauman $^{*}$, Donald T. Gavel ${ }^{\mathrm{b}}$, Richard G. Dekany ${ }^{\mathrm{c}}$, Brent L. Ellerbroek ${ }^{\mathrm{d}}$ \\ ${ }^{a}$ Lawrence Livermore National Laboratory, 7000 East Ave., M/S L-210, Livermore, CA 94550 \\ ${ }^{\mathrm{b}}$ UCO/Lick Observatory, UC Santa Cruz, 1156 High Street, Santa Cruz, CA 95064 \\ ${ }^{\text {c }}$ California Institute of Technology Optical Observatories, 1200 E. California Blvd., \\ Pasadena, CA 91125 \\ d Thirty-Meter Telescope (TMT) Project, 1200 E. California Blvd, MC 102-8, Pasadena, CA 91125
}

\begin{abstract}
We describe an exploratory optical design for the Narrow Field InfraRed Adaptive Optics (AO) System (NFIRAOS) Petite, a proposed adaptive optics system for the Thirty Meter Telescope Project. NFIRAOS will feed infrared spectrograph and wide-field imaging instruments with a diffraction limited beam. The adaptive optics system will require multi-guidestar tomographic wavefront sensing (WFS) and multi-conjugate AO correction. The NFIRAOS Petite design specifications include two small $60 \mathrm{~mm}$ diameter deformable mirrors (DM's) used in a woofer/tweeter or multiconjugate arrangement. At least one DM would be a micro-electromechanical system (MEMS) DM. The AO system would correct a 10 to 30 arcsec diameter science field as well as laser guide stars (LGS's) located within a 60 arcsec diameter field and low-order or tip/tilt natural guide stars (NGS's) within a 60 arcsec diameter field. The WFS's are located downstream of the DM's so that they can be operated in true closed-loop, which is not necessarily a given in extremely large telescope adaptive optics design. The WFS's include adjustable corrector elements which correct the static aberrations of the AO relay due to field position and LGS distance height.
\end{abstract}

Keywords: adaptive optics, NFIRAOS, Thirty Meter Telescope, deformable mirror, wavefront sensor, microelectromechanical system (MEMS), aberration correction, infrared, laser guide star, sodium beacon

\section{INTRODUCTION}

The NFIRAOS Petite was envisioned as a moderate field AO system that could be implemented with relatively inexpensive MEMS technologies. The NFIRAOS Petite design uses two $60 \mathrm{~mm}$ diameter DM's (including at least one MEMS DM) attaining a very small footprint on the Nasmyth platform between telescope focus and the three science instruments it feeds. NFIRAOS feeds two spectrometers with a 10 arcsecond diameter science field, the Infrared Imaging Spectrograph (IRIS) and the Near Infrared Echelle Spectrograph (NIRES); and an infrared imager with a 30 arcsecond diameter field, the Wide Field Infrared Camera (WIRC) ${ }^{1}$. The overall field of view of the AO relay is 120 arcseconds diameter to allow for natural guide stars and an array of laser guide stars.

\section{REQUIREMENTS}

The requirements for the NFIRAOS Petite system include the following:

- $\mathrm{f} / 15$ science output with real image

- $10-30$ arcsecond diameter science field

- Sodium beacon LGS's within a 60 arcsec diameter field centered on the science field; zenith angle $\leq 60^{\circ}$

- Natural stars within 120 arcsec diameter field for tip/tilt or low-order wavefront sensing

- Aberrations do not limit performance

- Science wavelength band: $0.8-5.0 \mu$

- Two $60 \mathrm{~mm}$ diameter MEMS deformable mirrors, used either as woofer/tweeter or multi-conjugate

- True closed-loop operation

*bauman3@1lnl.gov; phone 1925 423-6592; fax 1925 422-1796

Astronomical Adaptive Optics Systems and Applications II, edited by Robert K. Tyson, Michael Lloyd-Hart, Proceedings of SPIE Vol. 5903, 590303, (2005) $\cdot 0277-786$ X/05/\$15 $\cdot$ doi: $10.1117 / 12.618220$ 


\section{OPTICAL DESIGN}

The requirement of two pupil planes plus a final real image results in at least two powered mirrors in the AO relay, assuming that the DM's are not powered; this is dictated by first-order optics. The conventional AO relay design, where DM's are located in collimated space, would result in 4 powered optics: one OAP to collimate the light for the first DM; another pair of OAP's to re-collimate the light and form another pupil or a 10km position for the second DM; and one additional OAP to form a real image. By removing the collimated-space constraint, two warm surfaces can be eliminated in the AO relay. We would need just one relay optic before each DM to produce a pupil. The second relay optic would also produce a real image following the $2^{\text {nd }} \mathrm{DM}$. Since each relay optic is used at finite conjugates with real objects and images, the natural result is that each relay optic is an ellipsoid rather than a parabola.

The goal of using a $60 \mathrm{~mm}$ DM size is driven by two considerations: initial studies of pupil-aberration-induced anisoplanatism suggest that DM's of at least $60 \mathrm{~mm}$ are needed to avoid excessive anisoplanatism for a 30 arcsec field $^{2}$; and $60 \mathrm{~mm}$ is a feasible size for a MEMS DM, although not currently available. Requiring a larger DM would reduce the anisoplanatism, but at the cost of MEMS feasibility.

This system was designed for true closed-loop operation. Other possible choices include an open-loop mode where we would pick off guide star light before it strikes any DM, and correct the GS light and the science light with separate DM's. The advantage to this kind of configuration is that the relay need not be designed to pass the much larger GS field (1-2 arcmin diameter GS field vs. 10-30 arcsec diameter science field). The optics for the GS light would not need to image well a large field simultaneously, but only a number of very small fields centered about each guide star. The disadvantage, of course, is that the AO system would not operate in true closed-loop mode.

The GS light could be corrected in open-loop if the DM for the GS light can be characterized accurately enough. Another possibility is to use a monitor for the GS-path DM wherein an auxiliary source of collimated light is introduced at normal incidence to the DM and measured by a wavefront sensor or interferometer. One might describe this as quasiclosed-loop operation.

Nevertheless, a closed-loop design was chosen because it is a proven design concept which lowers technical risk.

\subsection{AO Relay Optical Design}

Figure 1 shows the optical layout. As described earlier, the adaptive optics relay consists of two off-axis ellipsoids that create two optical spaces for the DM's. In narrow-field mode, deformable mirrors are a woofer-tweeter pair, each placed conjugate to the ground $(0 \mathrm{~km}$ altitude). The design allows easy upgrade from narrow field to multi-conjugate mode by moving either of the deformable mirrors upstream by $40 \mathrm{~mm}$ to a $10 \mathrm{~km}$ conjugate position. It is recommended that the second DM be the $10 \mathrm{~km}$ conjugate mirror so that wavefront correction tends to undo the atmospherically induced ray deviations. 


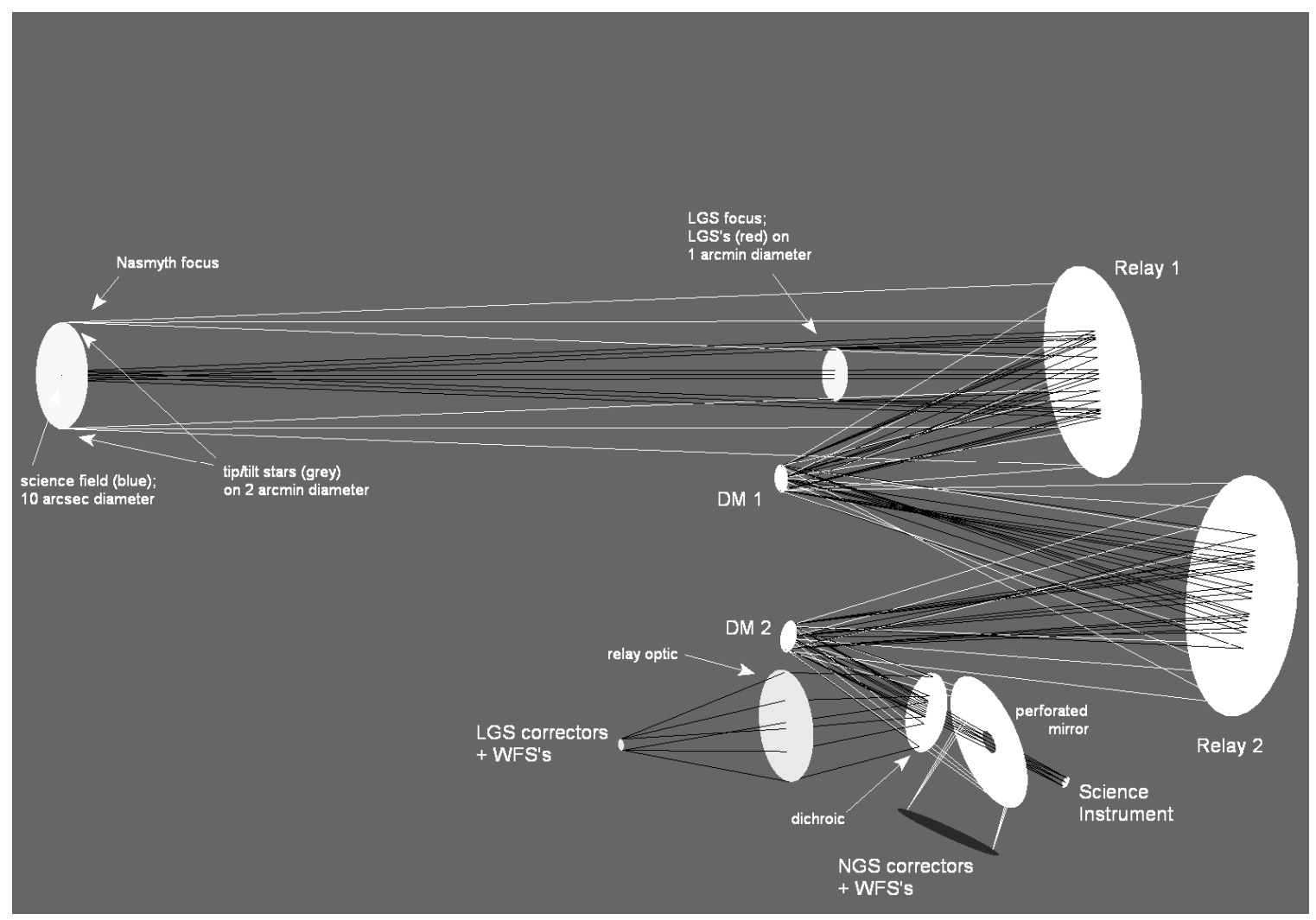

Figure 1: AO optical relay for NFIRAOS Petite. The optics shown fit in a space $1.5 \mathrm{~m}$ wide by $2 \mathrm{~m}$ long by $0.5 \mathrm{~m}$ high.

\subsection{Deformable Mirrors}

For narrow field mode, the deformable mirrors are a woofer-tweeter pair. The woofer can be a $60 \mathrm{~mm}$ bimorph mirror with at least 8 microns stroke (focus mode) and 36 degrees of freedom (this type of mirror is available from a number of manufacturers). The tweeter is a MEMS device with 100 actuators across the beam diameter; each actuator requires at least 1.5 microns stroke.

In multi-conjugate mode, the $0 \mathrm{~km}$ mirror will need to have at least 6 microns stroke. The $10 \mathrm{~km}$ mirror needs to have at least 80 actuators across and at least 1.5 microns stroke. The tweeter MEMS device that was used in narrow field mode could be reused for this purpose.

\subsection{Laser guidestar wavefront sensors}

An LGS wavelength dichroic beam splitter in the output beam picks off the LGS light and sends it to the high-order wavefront sensors. Since the LGS light is diverging in this space and it is necessary to provide a real image for pick-off mirrors (or other field selection mechanism) to the WFS's, it is necessary to provide a focusing element in the wavefront sensor path. This is accomplished with the air-spaced doublet shown. The wavefront sensors consist of the usual Hartmann setup: a lens following the focus to re-image the pupil, a lenslet array conjugate to the pupil/ground, and a relay to re-image the Hartmann spots to the wavefront sensor CCD.

The finite range of the LGS's results in a significant axial image shift compared to the infinity focus position of natural stars: approximately $2.2 \mathrm{~m}$ for an f/15 telescope with LGS's at $90 \mathrm{~km}$ range and $1.1 \mathrm{~m}$ for LGS's at $180 \mathrm{~km}$ range $\left(60^{\circ}\right.$ zenith angle). 


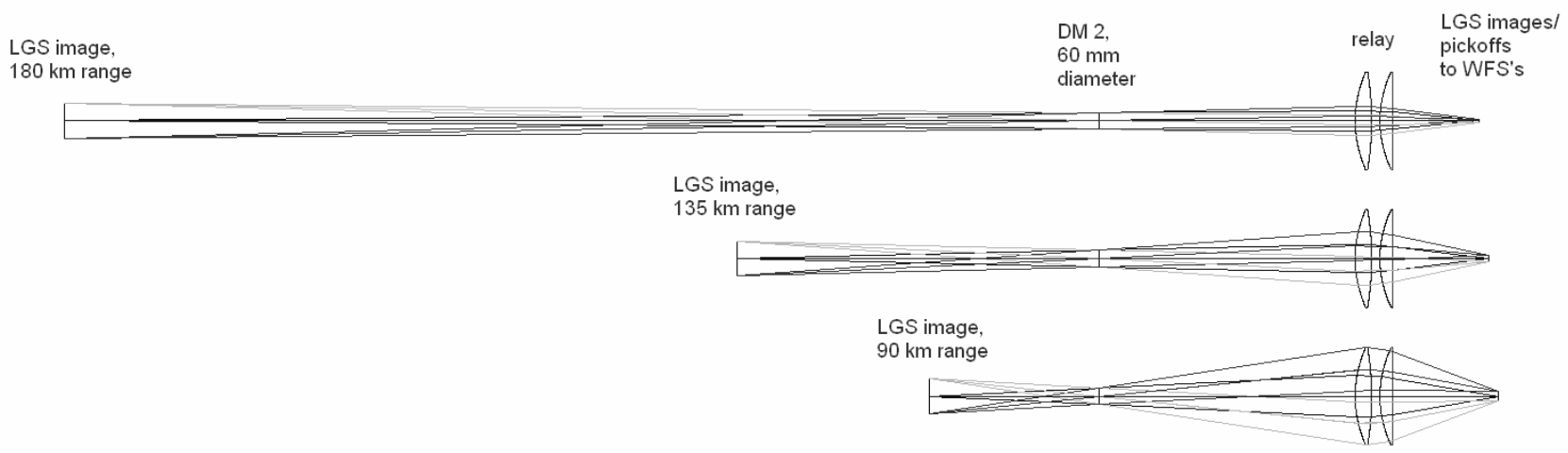

Figure 2: LGS re-imaging lens with LGS's of varying range. The illustrated beam begins in optical space following the last relay optic with varying f-numbers. Since the LGS beam is always diverging following the $2^{\text {nd }}$ DM, a relay lens is necessary to create a real image where the light can picked off and sent to WFS's. The relay lens consists of an air-spaced doublet.

The image space $\mathrm{f} \#$ of the LGS relay varies from $\mathrm{f} / 3$ to $\mathrm{f} / 5$, with a corresponding plate scale of $2.5-1.5 \mathrm{arcsec} / \mathrm{mm}$. There are two options for the pupil-imaging lens that follows the LGS focus and pick-off mirror. First, the pupilimaging lens need not collimate the light in general. Since the pupil is stationary even as the LGS range changes, keeping the air-spaced doublet, pupil-re-imaging lens, and lenslet array stationary keeps the beam size on the lenslet array, and hence the number of subapertures, constant. The penalty is that either the reference centroids for the wavefront sensor would need to be updated to reflect the changing LGS range, or that the relay optic between the lenslet array and the CCD would need to have variable magnification in order to accommodate the changing size of the Hartmann spot pattern. The other option is to design a zoom collimating lens which would accept the varying $\mathrm{f} \#$ of the incoming beam and produce a constant-size, collimated output.

\subsection{Aberration correctors}

The two off-axis ellipse design introduces up to 15 microns peak-to-valley of aberration for the GS light, consisting of mostly astigmatism. There are a few ways of handling this offset. 1) Astigmatism can be generated with an Alvarez lens pair $^{3}$ or with an axially translating cylindrical lens in non-collimated space. Coma can be generated by a generalized Alvarez lens pair ${ }^{4}$. 2) Another option is to put in a low order (no more than $4^{\text {th }}$ order radial is needed) deformable mirror in the path to the lenslet array. Because of the narrow field, it is not strictly necessary to place this $\mathrm{DM}$ at a pupil. It will be necessary to control the deformable mirror's figure very accurately since any inaccuracy will directly contribute to the AO system error budget. If the DM is not an accurate "go-to" MEMS device (for example if it is a bimorph made of piezo material, which has hysteresis), then the surface must be measured so it can be driven to the proper figure. A Fizeau type interferometer can be employed to do the measurement, looking normal to the reflective surface of the DM (see Figure 3). The control bandwidth needs be commensurate with the rate at which the relay aberrations change with changing LGS focus. Although this rate has not been thoroughly analyzed, we can estimate it as $\sim 20$ microns $/ 3$ hours $=10 \mathrm{~nm} / 11 \mathrm{sec}$, so an update every 10 seconds should suffice.

In addition, for the LGS light, the radial format CCD, being developed under an Adaptive Optics Development Program (AODP) grant to James Beletic and Jerry Nelson, is designed to measure Hartmann centroids linearly and accurately over a wide dynamic range. It may be sufficient to just subtract the known LGS wavefront offset in software from this measurement.

These represent significant, but certainly not insurmountable tasks in the optical design. 


\subsection{Natural Guide Star Tip/Tilt/Focus Sensors}

For narrow field mode, an annular mirror is placed at the output focal plane. This mirror has a 10 arcsecond diameter hole for the science field. In multi-conjugate (wide-field) mode, another annular mirror with a 30 arcsecond diameter hole is used. The outer diameter of the field picked off by the annular mirror is 120 arcseconds diameter. The light reflected from the annular mirror is fed to a field acquisition camera consisting of a re-imaging lens and a detector, for selecting guide stars. When the guide stars are selected, small deployable pickoff mirrors send the guide star light to the respective tip/tilt/focus sensors (see Figure 4). For each guide star, it will be necessary to use a second fold mirror that can tipped, tilted, and repositioned in order to equalize path length into these sensors and to position the pupil at a cold pupil stop. The repositioning could alternatively be accomplished by moving the detector and cold stop in $\mathrm{z}$ (focus).

In narrow field mode, the natural guide stars must be corrected for higher order anisoplanatic error in order to achieve high sky coverage. This is accomplished with a MEMS deformable mirror placed in the guidestar path after the pickoff. Again, because of the small field, it is not necessary to put the DM at a pupil. Assuming that the MEMS DM's will not be qualified to work at cryogenic temperatures or vacuum, they will be outside of the dewar as shown in Figure 3. We put the focusing mirror on a tip/tilt stage so that this reflection serves as the second steering mirror.

\subsection{Field Derotation}

It is envisioned that the NFIRAOS relay will remain fixed on the Nasmyth platform. It is desired to derotate the field before sending to the science instruments however. One method of accomplishing this is with a K-mirror in the diverging $\mathrm{f} / 15$ beam before the first off-axis ellipse, where there is plenty of room to put these large flats. This derotates the science field, field of natural tip/tilt/focus stars, and the constellation of laser guide stars. An alternative is to put separate derotaters at the output, at the feed to each science instrument. It will then be necessary to rotate the natural guide star sensors separately as well (probably by rolling it as a unit, in barrel fashion). This is probably a less preferable option since sensor motion must be extremely well coordinated with the science K-mirrors. The co-location in the focal planes must match to a fraction of the diffraction limit. Assuming 1 milli-arcsecond tolerance at $\mathrm{f} / 15$ this nutation accuracy would need to be plus or minus 1 micron.

\section{PERFORMANCE}

A summary of NFIRAOS "Petite" system performance is given in Table 1. Both the narrow and multi-conjugate wide field modes achieve the Science-Based Requirements Document (SRD) specifications. The use of deformable mirrors in the tip/tilt/focus sensor paths enables this design to achieve $90 \%$ sky coverage in narrow field mode as well as multiconjugate mode.

Including input and output windows and the $\mathrm{K}$ mirror derotator, the science path for the NFIRAOS Petite design has 10 optical surfaces. By keeping the system cooled to -31 degrees Celsius, the system emissivity meets the SRD requirement of no more than $15 \%$ of the telescope + inter $\mathrm{OH}$ line sky background (Table 2). With the conservative estimates of reflectivity and transmissivity shown in Table 2 , the AO relay throughput is $54 \%$. 


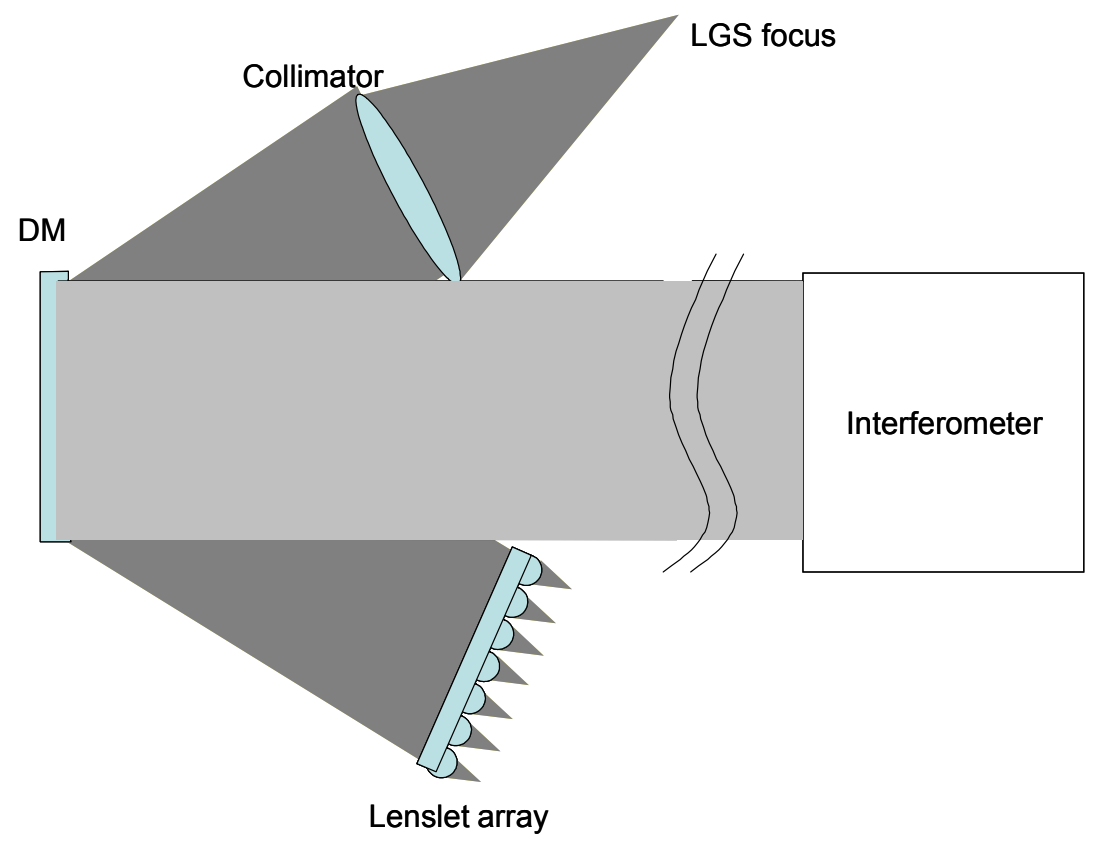

Figure 3. Using a MEMS deformable mirror in the wavefront sensor path to correct the AO relay's aberration of LGS light. An interferometer can probe the DM if the voltage response is uncertain or hysteretic.

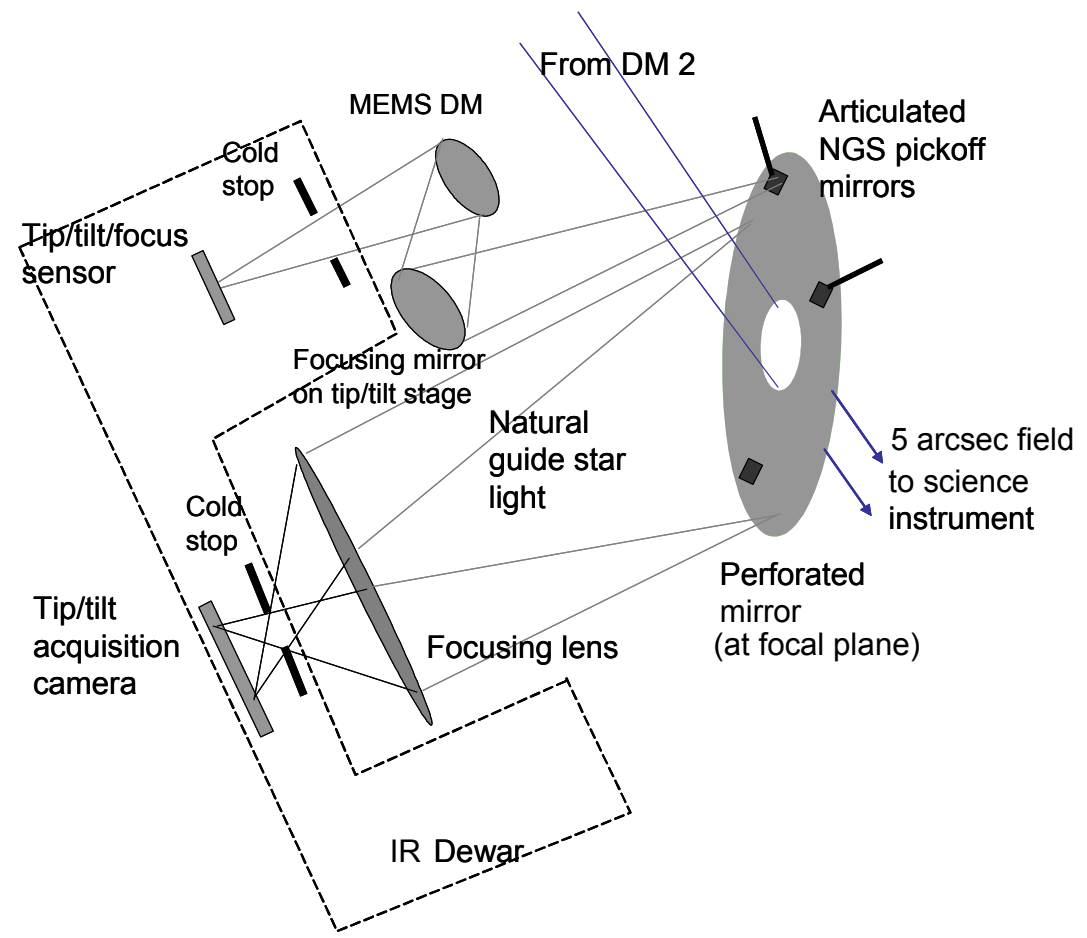

Figure 4. Natural guidestar pickoff and sensing concept. 
Table 1. NFIRAOS Petite Wavefront Error Budget.

\begin{tabular}{|c|c|c|c|c|c|c|c|}
\hline & \multicolumn{4}{|c|}{ Narrow field mode } & \multicolumn{3}{|c|}{ Multi-conjugate (wide field) mode } \\
\hline & WFE & Strehl & Strehl & & WFE & Strehl & Strehl \\
\hline & $\mathrm{nm}$ & $1 \mu$ & $2 \mu$ & & $\mathrm{nm}$ & $1 \mu$ & $2 \mu$ \\
\hline Higher Order WF & 193.27 & & & & 179.17 & & \\
\hline Fitting & 77.66 & 0.79 & 0.94 & & 82.20 & 0.77 & 0.94 \\
\hline Gen. Aniso (10 asec) & 144.05 & 0.44 & 0.81 & (30asec) & 121.55 & 0.56 & 0.86 \\
\hline Tomography & 83.52 & 0.76 & 0.93 & & 83.52 & 0.76 & 0.93 \\
\hline Meas & 40.00 & 0.94 & 0.98 & & 40.00 & 0.94 & 0.98 \\
\hline Bandwidth & 44.66 & 0.92 & 0.98 & & 44.66 & 0.92 & 0.98 \\
\hline Tip/Tilt & 0.00 & & & & 0.00 & & \\
\hline \multicolumn{8}{|l|}{ Tilt Aniso } \\
\hline \multicolumn{8}{|l|}{ Meas } \\
\hline \multicolumn{8}{|l|}{ Bandwidth } \\
\hline Total & 193.27 & 0.2289 & 0.69 & & 179.17 & 0.2816 & 0.73 \\
\hline Total, no aniso & 128.85 & 0.5192 & 0.85 & & 131.64 & 0.5045 & 0.84 \\
\hline Sky Coverage (Gal. pole) & & $90 \%$ & & & & $90 \%$ & \\
\hline \multicolumn{8}{|l|}{ Observing Parameters } \\
\hline Telescope diameter & $\mathrm{D}$ & 30 & $\mathrm{~m}$ & & & & \\
\hline Wavelength & $\lambda_{0}$ & 500 & $\mathrm{~nm}$ & & & & \\
\hline Coherence cell size & $r_{0}$ & 0.15 & $\mathrm{~m}$ & & & & \\
\hline Greenwood frequency & $f_{g}$ & 50 & $\mathrm{~Hz}$ & $\left(\tau_{0}=3 \mathrm{~ms}\right)$ & & & \\
\hline Controller frequency & $f_{c}$ & 100 & $\mathrm{~Hz}$ & & & & \\
\hline Outer scale & $\mathrm{L}_{0}$ & $>30$ & $\mathrm{~m}$ & & & & \\
\hline Turbulence Profile & $\mathrm{C}_{\mathrm{n}}{ }^{2}$ & $\begin{array}{l}\text { Cerro } \\
\text { Pachon }\end{array}$ & 7-layer & & & & \\
\hline Isoplanatic angle & $\theta_{0}$ & 2.45 & $\operatorname{arcsec}$ & & & & \\
\hline LGS constellation & $\Theta$ & 30 & $\operatorname{arcsec}$ & diam & & & \\
\hline $\begin{array}{l}\text { Number of guidestars } \\
\text { Science field }\end{array}$ & $\begin{array}{l}\mathrm{N}_{\mathrm{gs}} \\
\theta\end{array}$ & $\begin{array}{l}5 \\
10\end{array}$ & arcsec & diam & & & \\
\hline Number of DMs & $\mathrm{N}_{\mathrm{DM}}$ & 2 & & & & & \\
\hline Actuator spacing, DM 0 & $d$ & 0.30 & $\mathrm{~m}$ & & & & \\
\hline
\end{tabular}


Table 2. Throughput and emissivity. Emission units are photons $/ \mathrm{sec} / \mathrm{nm} / \operatorname{arcsec}^{2}$

\begin{tabular}{|c|c|c|c|c|c|c|}
\hline & & & Band & & & \\
\hline Mirror & $\mathrm{R}$ or $\mathrm{T}$ & Temperature & $1 \mu$ & $1.6 \mu$ & $2.2 \mu$ & $3 \mu$ \\
\hline Sky (inter $\mathrm{OH}$ ) & & & $2.00 \mathrm{E}+00$ & $1.00 \mathrm{E}+00$ & $2.00 \mathrm{E}+00$ & $4.00 \mathrm{E}+03$ \\
\hline Primary & 0.95 & 0 & $9.120 \mathrm{E}-12$ & 5.327E-04 & $1.188 \mathrm{E}+00$ & $2.042 E+02$ \\
\hline Secondary & 0.95 & 0 & $9.120 \mathrm{E}-12$ & 5.327E-04 & $1.188 \mathrm{E}+00$ & $2.042 \mathrm{E}+02$ \\
\hline Sub Total & 0.9025 & & $2.000 \mathrm{E}+00$ & $1.001 \mathrm{E}+00$ & $4.375 \mathrm{E}+00$ & $4.408 \mathrm{E}+03$ \\
\hline $15 \%$ SRD spec & & & $3.000 \mathrm{E}-01$ & $1.502 \mathrm{E}-01$ & $6.563 \mathrm{E}-01$ & $6.613 E+02$ \\
\hline AO Window & 0.95 & 0 & & & & \\
\hline OAE1 & 0.95 & -31 & 1.067E-14 & 7.835E-06 & $5.520 \mathrm{E}-02$ & $2.152 E+01$ \\
\hline DM1 & 0.9 & -31 & $2.133 \mathrm{E}-14$ & 1.567E-05 & 1.104E-01 & $4.304 E+01$ \\
\hline OAE2 & 0.95 & -31 & $1.067 \mathrm{E}-14$ & 7.835E-06 & $5.520 \mathrm{E}-02$ & $2.152 E+01$ \\
\hline DM2 & 0.9 & -31 & $2.133 \mathrm{E}-14$ & 1.567E-05 & 1.104E-01 & $4.304 \mathrm{E}+01$ \\
\hline Dichroic & 0.9 & -31 & $2.133 \mathrm{E}-14$ & 1.567E-05 & 1.104E-01 & $4.304 E+01$ \\
\hline Kmirror & 0.95 & -31 & 1.067E-14 & 7.835E-06 & $5.520 \mathrm{E}-02$ & $2.152 E+01$ \\
\hline Kmirror & 0.95 & -31 & 1.067E-14 & 7.835E-06 & $5.520 \mathrm{E}-02$ & $2.152 E+01$ \\
\hline Kmirror & 0.95 & -31 & $1.067 \mathrm{E}-14$ & 7.835E-06 & $5.520 \mathrm{E}-02$ & $2.152 E+01$ \\
\hline IR Window & 0.95 & -31 & $1.067 \mathrm{E}-14$ & 7.835E-06 & $5.520 \mathrm{E}-02$ & $2.152 E+01$ \\
\hline Sub Total & 0.54 & & $1.280 \mathrm{E}-13$ & $9.402 \mathrm{E}-05$ & 6.624E-01 & $2.582 E+02$ \\
\hline
\end{tabular}

\section{CONCLUSIONS}

An exploratory optical design for a TMT NFIRAOS Petite system has been described. The design uses 60mm DM's (at least one of which is a MEMS) in order to reduce costs. The optical design appears to be feasible and possesses measures to reduce optic count in the science field. A number of optical design issues related to off-axis aberrations in the very large guide star field have been enumerated, and a number of design options have been described. Performance appears to meet instrument requirements.

\section{ACKNOWLEDGEMENTS}

This work was performed under the auspices of the U.S. Department of Energy by University of California, Lawrence Livermore National Laboratory under Contract W-7405-Eng-48. This work was supported in part by the Gordon and Betty Moore Foundation through its grant to the UCO/Lick Laboratory for Adaptive Optics.

This paper was prepared as part of the work of the Thirty-Meter Telescope (TMT) Project. TMT is a partnership of the Association of Universities for Research in Astronomy (AURA), the Association of Canadian Universities for Research in Astronomy (ACURA), the California Institute of Technology, and the University of California. The partners gratefully acknowledge the support of the Gordon and Betty Moore Foundation, the U.S. National Science Foundation (NSF), the National Research Council of Canada, the Natural Sciences and Engineering Research Council of Canada, and the Gemini Partnership.

\section{REFERENCES}

1. http://www.astro.caltech.edu/observatories/tmt/NFIRAOS_RFP.pdf

2. B.J. Bauman, "Anisoplanatism in adaptive optics systems due to pupil aberrations", this conference.

3. L.W. Alvarez and W. E. Humphrey, "Variable-power lens and system," U. S. patent 3,507,565, 21 Apr. 1970.

4. I.A. Palusinski, J.M. Sasián, and J.E. Greivenkamp, "Lateral-shift variable aberration generators", Applied Optics, 38, 86-90, 1999. 